\title{
Residual Gauge Invariance in a Massive Lorentz-Violating Extension of QED
}

\author{
B. Alves Marques, ${ }^{1,2}$ A. P. Baêta Scarpelli, ${ }^{1}$ J. C. C. Felipe $\mathbb{D}^{1}{ }^{3}$ and L. C. T. Brito ${ }^{4}$ \\ ${ }^{1}$ Centro Federal de Educação Tecnológica, MG Avenida Amazonas, 7675-30510-000-Nova Gameleira-Belo Horizonte-MG, Brazil \\ ${ }^{2}$ Instituto Federal de Minas Gerais, Campus Sabará Rodovia MGC, 262 Sobradinho, Minas Gerais, Brazil \\ ${ }^{3}$ Instituto de Engenharia, Ciência e Tecnologia, Universidade Federal dos Vales Do Jequitinhonha e Mucuri Avenida Um, no. 4050, \\ 39447-814, Cidade Universitária, Janaúba, MG, Brazil \\ ${ }^{4}$ Universidade Federal de Lavras, Departamento de Fsica Caixa Postal 3037, 37., 200-000 Lavras, Minas Gerais, Brazil \\ Correspondence should be addressed to J. C. C. Felipe; jean.cfelipe@ufvjm.edu.br
}

Received 28 July 2021; Revised 16 December 2021; Accepted 1 February 2022; Published 12 February 2022

Academic Editor: Enrico Lunghi

Copyright (c) 2022 B. Alves Marques et al. This is an open access article distributed under the Creative Commons Attribution License, which permits unrestricted use, distribution, and reproduction in any medium, provided the original work is properly cited. The publication of this article was funded by SCOAP S. $^{3}$

\begin{abstract}
We reassess an alternative CPT-odd electrodynamics obtained from a Palatini-like procedure. Starting from a more general situation, we analyze the physical consistency of the model for different values of the parameter introduced in the mass tensor. We show that there is a residual gauge invariance in the model if the local transformation is taken to vary only in the direction of the Lorentz-breaking vector. This residual gauge invariance can be extended to all models whose only source of gauge symmetry breaking is such a mass term.
\end{abstract}

\section{Introduction}

It has been more than two decades that the investigation of Lorentz-violating models got the attention of the community of quantum field theory physicists. Indeed, it is believed that the usual symmetries of special relativity will be broken in the limit of very high energy physics as an effect of quantum gravity issues [1-5]. This possibility was first discussed in the papers of Kostelecký and Samuel $[1,2]$, but it gained great dimension when Carroll, Field, and Jackiw proposed a modification of the classical electrodynamics by the inclusion of a Chern-Simons-like term in the photon sector [6]. This term violates both CPT and Lorentz symmetries and gives rise to a wide range of interesting physical effects, which were intensively studied. Many papers treated features of the model like its physical consistency $[7,8]$, the possibility of the CFJ term being radiatively generated [9-19], and many other aspects [20-27].

Further, a general description of Lorentz violation in quantum field theories was provided by the Standard Model Extension (SME) [28-31]. The SME, which includes the
Carroll-Field-Jackiw (CFJ) term, establishes a set of constant tensors as the parameters of Lorentz violation, whose small magnitudes are strongly constrained by experiments [32]. These constant tensors would arise from spontaneous Lorentz symmetry breaking at very high energies [28]. It is important to note that the SME preserves $\mathrm{SU}(3) \times \mathrm{SU}(2) \times$ $U(1)$ gauge symmetry and the renormalizability.

One aspect that has been gaining increasing attention is the inclusion of massive photons in models that violate Lorentz symmetry. In general, it is believed that the fact that photons are massless is a direct consequence of gauge invariance of quantum electrodynamics (QED). Nevertheless, many studies of the physics beyond the Maxwell electromagnetism have been performed, mainly in the context of Proca model [33-37]. Although studies using astrophysical sources and laboratory experiments put very strong constraints in the photon mass [38-51], it may be possible that it has a very tiny but nonvanishing rest mass $m_{\gamma}$. It is interesting to note that the usual CFJ model already accommodates an effective mass for the photon which is proportional to the absolute value of the Lorentz-breaking vector $[52,53]$. Still, in the 
context of electrodynamics with the Carroll-Field-Jackiw term, an explicit mass term for the photon was used to repair unitarity problems for a time-like background vector [54].

On the other hand, models with Lorentz-violating (LVI) mass terms present remarkable peculiarities, like the ones pointed out in $[55,56]$, in which the gauge field has two massive degrees of freedom but the static force between charged particles is Coulomb-like. Many aspects like the quantum induction of Lorentz-breaking mass terms for the photon [57], the construction of a Stueckelberg Lagrangian in a generalized $R_{\xi}$ gauge [58], and spontaneous gauge symmetry breaking in a Lorentz-violating gauge-Higgs model [59] were investigated. In [60], some aspects of this kind of gauge symmetry breaking were focused in a study of dual models.

An alternative way of generating Lorentz-violating mass terms was investigated in [61], in which a Palatini-like formulation of the CFJ model was performed. Interestingly, this formulation, with the participation of the CFJ term, caused the emergence of an unusual mass contribution in the photon Lagrangian density. While the usual Proca mass term breaks only gauge invariance, the present mass contribution breaks Lorentz symmetry and, supposedly, gauge invariance. These results looked intriguing, since the model from which we begin, in which $F_{\mu \nu}$ is treated as an independent field, has no any evidence of violation of gauge symmetry. In this paper, we study a more general version of the massive model obtained in [61] and show that there is a residual gauge invariance in the model obtained from the Palatini-like formulation.

The paper is organized as follows: in Section 2, general discussions on the model are presented and the gauge field propagator is obtained; in Section 3, we carry out an analysis of the field equations; we study tree-level unitarity of the model in Section 4; in Section 5, we investigate the presence of a residual gauge invariance in the massive model; conclusions and final remarks are presented in Section 6.

\section{General Discussions on the Model}

Let us consider the following Lagrangian density of a Lorentz-breaking extension of spinorial quantum electrodynamics (QED),

$$
\begin{aligned}
L= & -\frac{1}{4} F_{\mu \nu} F^{\mu \nu}+\alpha \mu \kappa_{\mu} A_{v} \partial_{\beta} A_{\sigma} \varepsilon^{\mu \nu \beta \sigma}+\frac{1}{2} \mu^{2} A^{\mu} M_{\mu \nu} A^{v} \\
& -J_{\mu} A^{\mu}+\mathscr{L}_{F},
\end{aligned}
$$

with

$$
M_{\mu \nu}=\kappa^{2} \eta_{\mu \nu}-\rho \kappa_{\mu} \kappa_{v}
$$

in which $\alpha$ and $\rho$ are dimensionless constants, $\mu$ is a mass parameter and $\kappa^{\mu}$ is a background constant pseudovector that causes the violation of Lorentz symmetry. Besides, $F_{\mu \nu}=\partial_{\mu} A_{\nu}-\partial_{\nu} A_{\mu}, J^{\mu}=e \bar{\psi} \gamma^{\mu} \psi$ is the conventional matter current and $\mathscr{L}_{F}$ is the pure fermionic Lagrangian density. The gauge invariance of the model is apparently explicitly broken due to the presence of the mass term, (1/2) $\mu^{2} A^{\mu}$ $M_{\mu \nu} A^{v}$, which also includes a Lorentz-breaking part. It is worth to note that such a term is usually called nonminimal, since it accommodates nonminimal couplings to the curvature in field theories in curved space-time [62-65].

This particular LVI extension of QED, in which the background vector of the Carroll-Field-Jackiw (CFJ) term is responsible for the mass tensor, was obtained in [61], with $\alpha=-1, \rho=1$, and $\mu^{2}=1$. This was accomplished through a Palatini-type procedure, in which the $F^{\mu \nu}$ tensor and the gauge field were initially considered to be independent. The result was an unusual situation, in which the breaking of Lorentz's invariance induces a violation, at least apparent, of the gauge symmetry.

The breaking of gauge symmetry may cause problems to the consistency of the theory, as the violation of unitarity, with deleterious effects in the renormalizability of the model. A common procedure in cases like that is the searching for a hidden gauge symmetry, like the solution of Stueckelberg $[66,67]$, in which a mixed term that includes a scalar field is added to the Lagrangian density, such that gauge symmetry is restored. It is noteworthy that the idea of the Stueckelberg mechanism was extended to the Standard Model to give mass to the physical photon through the hypercharge $U(1)_{Y}$ $[68,69]$. Another approach is the application of a dualization method in order to obtain a gauge theory which describes the same physics as the original model. In both cases, it is assumed that the model of interest is a gaugefixed version of a gauge theory. We will perform further investigations on the possibility of finding a hidden gauge symmetry in the present model. Let us first analyze general features of the model (1).

The quadratic part of the Lagrangian density in the vectorial field $A^{\mu}$ can be written as

$$
\mathscr{L}_{G}=\frac{1}{2} A^{\mu} O_{\mu \nu} A^{v}
$$

with

$$
O_{\mu \nu}=\left(\square+\mu^{2} \kappa^{2}\right) \theta_{\mu \nu}+\mu^{2} \kappa^{2} \omega_{\mu \nu}-2 \alpha \mu S_{\mu \nu}-\rho \mu^{2} \Lambda_{\mu \nu}
$$

where $\theta_{\mu \nu}=\eta_{\mu \nu}-\partial_{\mu} \partial_{\nu} / \square$ and $\omega_{\mu \nu}=\partial_{\mu} \partial_{\nu} / \square$ are the transversal and longitudinal spin projectors, respectively, and the operators

$$
S_{\mu \nu}=\varepsilon_{\mu \nu \alpha \beta} \kappa^{\alpha} \partial^{\beta} \text { and } \Lambda_{\mu \nu}=\kappa_{\mu} \kappa_{v}
$$

are dependent on the background vector $\kappa^{\mu}$. The wave operator $O_{\mu \nu}$ is invertible. However, as we intend to define a closed algebra which includes the operators $\theta_{\mu \nu}, \omega_{\mu \nu}, \Lambda_{\mu \nu}$, and $S_{\mu \nu}$, it is necessary to include new operators, as Equation (6) indicates. It should be noted that no new term, such as a gauge-fixing, is being added to the wave operator. Since we 
have

$$
S_{\mu \alpha} S_{v}^{\alpha}=\left(\kappa^{2} \square-\lambda^{2}\right) \theta_{\mu \nu}-\lambda^{2} \omega_{\mu \nu}+\lambda\left(\Sigma_{\mu \nu}+\Sigma_{\nu \mu}\right)-\square \Lambda_{\mu \nu} \equiv f_{\mu \nu},
$$

with

$$
\Sigma_{\mu \nu}=\kappa_{\mu} \partial_{v}, \lambda \equiv \Sigma_{\mu}^{\mu}=\kappa_{\mu} \partial^{\mu}
$$

we include the operators $\Sigma_{\mu \nu}$ and $\Sigma_{v \mu}$ in the algebra, which is displayed in Table 1.

We thus obtain, after a straightforward but lengthy calculation, the vector field propagator, $\left\langle A_{\mu} A_{\nu}\right\rangle=i\left(O^{-1}\right)_{\mu \nu}$, in momentum-space:

$$
\begin{aligned}
\left\langle A_{\mu} A_{\nu}\right\rangle= & \frac{i}{D}\left\{-\left(p^{2}-\mu^{2} \kappa^{2}\right) \theta_{\mu \nu}+\frac{1}{\mu^{2} \kappa^{2}}\left[D+\frac{\lambda^{2}}{\tilde{D}}\left(p^{2} H+4 \alpha^{2} \mu^{2} \tilde{D}\right)\right] \omega_{\mu \nu}\right. \\
& \left.+2 i \alpha \mu S_{\mu \nu}-\frac{\lambda H}{\tilde{D}}\left(\Sigma_{\mu \nu}+\Sigma_{\nu \mu}\right)-\frac{\mu^{2}}{\tilde{D}}\left(-\kappa^{2} H+4 \alpha^{2} \tilde{D}\right) \Lambda_{\mu \nu}\right\},
\end{aligned}
$$

with

$$
\begin{gathered}
D=\left(p^{2}-\mu^{2} \kappa^{2}\right)^{2}+4 \alpha^{2} \mu^{2}\left(\kappa^{2} p^{2}-\lambda^{2}\right), \\
\tilde{D}=-\left(\kappa^{2} p^{2}-\rho \lambda^{2}\right)+\mu^{2} \kappa^{4}(1-\rho), \\
H=-\rho\left(p^{2}-\mu^{2} \kappa^{2}\right)+4 \alpha^{2} \mu^{2} \kappa^{2}(1-\rho) .
\end{gathered}
$$

The operators, in momentum-space, are given by

$$
\begin{gathered}
\theta_{\mu \nu}=\eta_{\mu \nu}-\omega_{\mu \nu}, \omega_{\mu \nu}=\frac{p_{\mu} p_{v}}{p^{2}}, S_{\mu \nu}=\varepsilon_{\mu \nu \alpha \beta} \kappa^{\alpha} p^{\beta}, \\
\Sigma_{\mu \nu}=\kappa_{\mu} p_{\nu} \text { and } \Lambda_{\mu \nu}=\kappa_{\mu} \kappa_{v} .
\end{gathered}
$$

We recognize above the propagator for the model of [61], if we fix $\alpha=-1, \rho=1$, and $\mu^{2}=1$. Interesting that the dispersion relations extracted from the denominator $D$, responsible for the massive modes, are not affected by the constant $\rho$. This parameter only influences the modes coming from $\tilde{D}$. In [61], the physical consistency of the modes of propagation of the vector field was studied in detail. For now, let us study the effects of the constant $\rho$ on the model and, for comparison, let us fix $\alpha=-1$ and $\mu^{2}=1$.

\section{Field Equations}

Since in [61], it was shown that only a spacelike $\kappa^{\mu}$ produces meaningful modes of propagation of the vector field, we restrict ourselves here to the analysis of this case. Let us consider a referential frame in which we have a purely spacelike background vector given by $\kappa^{\mu}=(0, \vec{\kappa})$, with $|\vec{\kappa}|^{2}=t^{2}$, and choose the direction of the $z$-axis as the one towards which the $\vec{\kappa}$ vector is pointed. So, we have the poles

$$
p_{0}^{2}=p_{\perp}^{2}+\left(p_{z}+t\right)^{2} \equiv m_{+}^{2} \text { and } p_{0}^{2}=p_{\perp}^{2}+\left(p_{z}-t\right)^{2} \equiv m_{-}^{2},
$$

due to the denominator $D$, and

$$
p_{0}^{2}=p_{\perp}^{2}+(1-\rho)\left(p_{z}^{2}-t^{2}\right) \equiv \tilde{m}^{2}
$$

due to the denominator $\tilde{D}$, where $\vec{p}_{\perp}$ is the component of $p$ orthogonal to the $z$-axis.

We first examine the solutions of the field equation in momentum-space,

$$
\left(-p^{2}+\kappa^{2}\right) A_{\mu}+(p \cdot A) p_{\mu}-\rho(\kappa \cdot A) \kappa_{\mu}+2 i \varepsilon_{\mu \nu \alpha \beta} A^{v} \kappa^{\alpha} p^{\beta}=0,
$$

with $A^{\mu}=(\phi, A)$. The contraction of the above equation with $p^{\mu}$ gives us the gauge condition $(p \cdot A)=\left(\rho \lambda / \kappa^{2}\right)(\kappa \cdot A)$ , which, after the substitution in (14), furnishes us

$$
\left(-p^{2}+\kappa^{2}\right) A_{\mu}+\rho\left(\frac{\lambda}{\kappa^{2}} p_{\mu}-\kappa_{\mu}\right)(\kappa \cdot A)+2 i \varepsilon_{\mu \nu \alpha \beta} A^{v} \kappa^{\alpha} p^{\beta}=0,
$$

in which $\lambda=\Sigma_{\mu}^{\mu}$, according to the definitions of (10) for the operators in momentum-space. For our spacelike $\kappa^{\mu}$, we have

$$
-\left(p^{2}+t^{2}\right) A_{\mu}-\rho\left(p_{z} p_{\mu}+t^{2} \delta_{\mu}^{3}\right) A_{z}+2 i t \varepsilon_{\mu \nu 3 \beta} A^{v} p^{\beta}=0
$$

In addition, let us define the $x$-axis as the direction aligned with the component of $A$ which is orthogonal to $\vec{\kappa}$, so that $A_{y}=0$. For the $z$-component of the field equation, we have

$$
\left[p_{0}^{2}-p_{\perp}^{2}-(1-\rho)\left(p_{z}^{2}-t^{2}\right)\right] A_{z}=0
$$

We see that the pole $p_{0}^{2}=\tilde{m}^{2}$ automatically satisfies the equation above. For $A_{z} \neq 0$, the poles $p_{0}^{2}=m_{ \pm}^{2}$ are constrained to equal $\tilde{m}^{2}$, which means, for a nonnull $\rho$,

$$
\begin{aligned}
\left(p_{z}+t\right)^{2} & =(1-\rho)\left(p_{z}^{2}-t^{2}\right) \Rightarrow p_{z} \\
& =\left\{\begin{array}{ll}
-t, & \text { if } 0<\rho 1 \\
\left(\frac{\rho-2}{\rho}\right) t, & \text { if } \rho>1 \text { or } \rho<0
\end{array}, \text { for } \tilde{m}^{2}=m_{+}^{2},\right. \\
\left(p_{z}-t\right)^{2} & =(1-\rho)\left(p_{z}^{2}-t^{2}\right) \Rightarrow p_{z} \\
& =\left\{\begin{array}{ll}
t, & \text { if } 0<\rho 1 \\
\left(\frac{2-\rho}{\rho}\right) t, & \text { if } \rho>1 \text { or } \rho<0
\end{array}, \text { for } \tilde{m}^{2}=m_{-}^{2} .\right.
\end{aligned}
$$

It is not possible that these two poles satisfy these 
TABLE 1: Multiplicative table. The products are supposed to obey the order "row times column."

\begin{tabular}{|c|c|c|c|c|c|c|}
\hline & $\theta_{v}^{\alpha}$ & $\omega_{v}^{\alpha}$ & $S_{v}^{\alpha}$ & $\Lambda_{v}^{\alpha}$ & $\sum_{v}^{\alpha}$ & $\sum_{v}^{\alpha}$ \\
\hline$\theta_{\mu \alpha}$ & $\theta_{\mu \nu}$ & 0 & $S_{\mu \nu}$ & $\Lambda_{\mu \nu}-(\lambda / \square) \Sigma_{\nu \mu}$ & $\Sigma_{\mu \nu}-\lambda \omega_{\mu \nu}$ & 0 \\
\hline$\omega_{\mu \alpha}$ & 0 & $\omega_{\mu \nu}$ & 0 & $(\lambda / \square) \Sigma_{v \mu}$ & $\lambda \omega_{\mu \nu}$ & $\Sigma_{v \mu}$ \\
\hline$S_{\mu \alpha}$ & $S_{\mu \nu}$ & 0 & $f_{\mu \nu}$ & 0 & 0 & 0 \\
\hline$\Lambda_{\mu \alpha}$ & $\Lambda_{\mu \nu}-(\lambda / \square) \Sigma_{\mu \nu}$ & $(\lambda / \square) \Sigma_{\mu \nu}$ & 0 & $\kappa^{2} \Lambda_{\mu \nu}$ & $\kappa^{2} \Sigma_{\mu \nu}$ & $\lambda \Lambda_{\mu \nu}$ \\
\hline$\Sigma_{\mu \alpha}$ & 0 & $\Sigma_{\mu \nu}$ & 0 & $\lambda \Lambda_{\mu \nu}$ & $\lambda \Sigma_{\mu \nu}$ & $\square \Lambda_{\mu \nu}$ \\
\hline$\Sigma_{\alpha \mu}$ & $\Sigma_{\nu \mu}-\lambda \omega_{\mu \nu}$ & $\lambda \omega_{\mu \nu}$ & 0 & $\kappa^{2} \Sigma_{v \mu}$ & $\kappa^{2} \square \omega_{\mu \nu}$ & $\lambda \Sigma_{v \mu}$ \\
\hline
\end{tabular}

conditions at the same time for an arbitrary $\rho$, if $t \neq 0$. The other equations of motion for $A_{z} \neq 0$, with $p_{0}^{2}=\tilde{m}^{2}$ and, consequently, $p^{2}+t^{2}=-\rho\left(p_{z}^{2}-t^{2}\right)$, are written as

$$
\left\{\begin{array}{l}
-\rho\left(p_{z}^{2}-t^{2}\right) A_{x}+\rho p_{x} p_{z} A_{z}+2 i t p_{y} \phi=0, \\
\rho p_{y} p_{z} A_{z}+2 i t\left(p_{0} A_{x}-p_{x} \phi\right)=0, \\
\rho\left(p_{z}^{2}-t^{2}\right) \phi-\rho p_{0} p_{z} A_{z}-2 i t p_{y} A_{x}=0 .
\end{array}\right.
$$

We try a solution with $\phi=0$, for which, after some manipulation of equations (19), we obtain

$$
\left(p_{0}^{2}-p_{y}^{2}\right) A_{x}=0
$$

If $A_{x} \neq 0$, we are left with the constraints $p_{x}=0$ and $p_{z}$ $= \pm t$, which correspond to the two modes $\tilde{m}^{2}=m_{+}^{2}$ $\left(p_{z}=-t\right)$ and $\tilde{m}^{2}=m_{-}^{2}\left(p_{z}=t\right)$. For the first mode, the electromagnetic field is polarized as $A=\left(A_{x}, 0, A_{z}\right)$ and propagates in the direction $p=\left(0, p_{y},-t\right)$, such that $p \cdot A=-t A_{z}$ and $p \cdot A=t A_{z}$. The gauge condition, however, imposes one more restriction:

$$
(p \cdot A)=\frac{\rho \lambda}{\kappa^{2}}(\kappa \cdot A) \Rightarrow(p \cdot A)=\rho t A_{z}
$$

So, this solution fixes $\rho=1$. For the mode with $\tilde{m}^{2}=m_{-}^{2}$, the conclusions are the same, only with the change in the sign of the $p_{z}$ component.

If $A_{x}=0$, taking into account the other equations, we necessarily have $p_{z}=0$, such that $\tilde{m}^{2}=p_{\perp}^{2}-(1-\rho) t^{2}$. Since, the poles $m_{ \pm}^{2}$ satisfy the 3-component of field equations for $p_{z}= \pm(\rho-2) t / \rho$, we have that, if $\rho=2$, the three poles are reduced to one $p_{0}^{2}=\tilde{m}^{2}=m_{ \pm}^{2}=p_{\perp}^{2}+t^{2}$, with the possible nonphysical consequences of a multiple pole.

In the case $A_{z}=0$, the mode $p_{0}^{2}=\tilde{m}^{2}$ is not excluded. It is not possible, however, to fix $\phi=0$. In order to obtain a solution which is valid for all values of $\rho$, we obtain the constrainings $p_{y}=0$ and $p_{z}= \pm t$, which satisfy one of the two conditions: $p_{0}^{2}=\tilde{m}^{2}=m_{+}^{2}$ or $p_{0}^{2}=\tilde{m}^{2}=m_{-}^{2}$.

In summary, in this section, we investigated how the solutions of the field equations are affected by the poles of the model. In the next section, we will look at the physical nature of these poles subject to specific conditions imposed by the field equations.

\section{Tree-Level Unitarity Analysis}

It is enlightening that we make an analysis of the physical nature of the poles. With this purpose, we investigate the tree-level unitarity of the model. It can be investigated through the propagator, when saturated by conserved currents,

$$
\mathcal{S P}=J^{\mu}\left\langle A_{\mu} A_{\nu}\right\rangle J^{* v}
$$

The current conservation in momentum-space is written as $p_{\mu} J^{\mu}=0$, such that

$$
\begin{gathered}
p \cdot J=p_{0} J_{0}-p \cdot J=0 \Rightarrow J_{0}=\frac{p \cdot J}{p_{0}} \\
J^{\mu} J_{\mu}^{*}=\left|J_{0}\right|^{2}-\left|J_{\perp}\right|^{2}-\left|J_{z}\right|^{2}=\frac{1}{p_{0}^{2}}\left[|p \cdot J|^{2}-p_{0}^{2}\left(\left|J_{\perp}\right|^{2}+\left|J_{z}\right|^{2}\right)\right] .
\end{gathered}
$$

Unitarity requires that the imaginary part of the residue of the saturated propagator in a physical pole is nonnegative (see $[70,71])$. This requirement can be checked by calculating the residue matrix in the pole for the complete propagator and, then, verifying if its eigenvalues are nonnegative. Here, we opt to analyze directly the saturated propagator. Note that in [70], this technique of analysis was used in a model obtained from the dimensional reduction of the CPT-even sector of the Standard Model Extension.

Turning our attention to the saturated propagator, in consequence of current conservation, only terms on $\eta_{\mu \nu}$ and $\Lambda_{\mu \nu}$ remain, such that, for our spacelike $\kappa^{\mu}$, we stay with

$$
\begin{aligned}
\mathcal{S P}= & \frac{i}{p_{0}^{2} D}\left\{-\left(p^{2}+t^{2}\right)\left[|p \cdot J|^{2}-p_{0}^{2}\left|J_{\perp}\right|^{2}\right]\right. \\
& \left.+\frac{p_{0}^{2}\left|J_{z}\right|^{2}}{\tilde{D}}\left[-t^{4} H+\left(p^{2}-3 t^{2}\right) \tilde{D}\right]\right\},
\end{aligned}
$$


with

$$
\begin{gathered}
D=\left(p_{0}^{2}-m_{+}^{2}\right)\left(p_{0}^{2}-m_{-}^{2}\right), \\
\tilde{D}=t^{2}\left(p_{0}^{2}-\tilde{m}^{2}\right), \\
H=-\rho\left(p^{2}+t^{2}\right)-4 t^{2}(1-\rho) .
\end{gathered}
$$

We have some potential problematic situations to analyze here: when $p_{z}= \pm t$, we have, in principle, double poles, with $\tilde{m}^{2}=m_{+}^{2}$ and $\tilde{m}^{2}=m_{-}^{2}$, respectively; this situation repeats when $p_{z}= \pm(\rho-2 / \rho) t$, with an additional complication if $\rho=2$, with $p_{z}=0$, which, at first sight, brings us a triple pole $\left(\tilde{m}^{2}=m_{+}^{2}=m_{-}^{2}\right)$. We study below these cases.

4.1. The Cases $p_{z}= \pm t$. Since these two cases are similar, we analyze here the referential frame in which $p_{z}=-t$, such that we stay with the poles $p_{0}^{2}=\tilde{m}^{2}=m_{+}^{2}=p_{\perp}^{2}$ and $p_{0}^{2}=m_{-}^{2}=p_{\perp}^{2}$ $+4 t^{2}$. This situation is possible both for $A_{z}=0$ and $A_{z} \neq 0$, as we verified in the field equations. The key point in order to analyze the possibility of a double pole is the fact that $p^{2}$ $+t^{2}=p_{0}^{2}-\tilde{m}^{2}$ in this referential frame. We stay with

$$
\mathcal{S P}=\frac{i}{p_{0}^{2}\left(p_{0}^{2}-\tilde{m}^{2}-4 t^{2}\right)}\left\{-\left[|p \cdot J|^{2}-p_{0}^{2}\left|J_{\perp}\right|^{2}\right]+\frac{p_{0}^{2}\left|J_{z}\right|^{2}}{\left(p_{0}^{2}-\tilde{m}^{2}\right)^{2}}\left[\left(p_{0}^{2}-\tilde{m}^{2}\right)\left(p_{0}^{2}-\tilde{m}^{2}+(\rho-4) t^{2}\right)+4 t^{4}(1-\rho)\right]\right\},
$$

and we see that, in order to eliminate the double pole, we need to have $\rho=1$, in accordance with the result we obtained in the study of the field equations in conjunction with the gauge condition. If we fix $\rho=1$, the saturated propagator reads

$\mathcal{S P}=\frac{i}{p_{0}^{2}\left(p_{0}^{2}-\tilde{m}^{2}-4 t^{2}\right)}\left\{-\left[|p \cdot J|^{2}-p_{0}^{2}\left|J_{\perp}\right|^{2}\right]+\frac{p_{0}^{2}\left|J_{z}\right|^{2}}{\left(p_{0}^{2}-\tilde{m}^{2}\right)}\left(p_{0}^{2}-\tilde{m}^{2}-3 t^{2}\right)\right\}$.

We are now in position to calculate the imaginary part of the residue in the two remaining poles. For the pole $p_{0}^{2}=\tilde{m}^{2}$, the calculation is straightforward and gives

$$
\operatorname{Im}\left[\mathscr{R}_{p_{0}^{2}=\tilde{m}^{2}}(\mathcal{S} \mathscr{P})\right]=\frac{3}{4}\left|J_{z}\right|^{2} .
$$

For the residue in the pole $p_{0}^{2}=m_{-}^{2}$, the term in $|p \cdot J|^{2}$ contributes. The residue gives us
We then remember that, for $p_{z}=-t$, we have $p_{x}=0$ and that, in this case, $\tilde{m}^{2}=p_{y}^{2}$ to obtain, after some calculation,

$$
\operatorname{Im}\left[\mathscr{R}_{p_{0}^{2}=p_{y}^{2}+4 t^{2}}(\mathcal{S P})\right]=\frac{\left|p_{y} J_{z}+4 t J_{y}\right|^{2}}{4\left(p_{y}^{2}+4 t^{2}\right)}+\left|J_{x}\right|^{2}>0 .
$$

The result above, which can be diagonalized, indicates a massive mode with two degrees of freedom.

4.2. The Cases $p_{z}= \pm(\rho-2 / \rho) t$. We here analyze the case $p_{z}=(\rho-2 / \rho) t$, since the two situations are similar. In this case, we have a possible double pole at $\operatorname{Im}\left[\mathscr{R}_{p_{0}^{2}=p_{\perp}^{2}+4 t^{2}}(\mathcal{S P P})\right]=\frac{1}{4\left(\tilde{m}^{2}+4 t^{2}\right)}\left\{-4|p \cdot J|^{2}+4\left(\tilde{m}^{2}+4 t^{2}\right)\left|J_{\perp}\right|^{2}+\left(\tilde{m}^{2}+4 t^{2}\right)\left|J_{z}\right|^{2}\right\}$.

$$
p_{0}^{2}=\tilde{m}^{2}=m_{+}^{2}=p_{\perp}^{2}+\frac{4(1-\rho)^{2} t^{2}}{\rho^{2}},
$$

and, if $\rho=2$, a triple pole. The saturated propagator, after algebraic manipulations, is given by

$$
\mathcal{S} \mathscr{P}=\frac{i}{p_{0}^{2}\left(p_{0}^{2}-\tilde{m}^{2}\right)\left[p_{0}^{2}-\tilde{m}^{2}-4(2-\rho) t^{2} / \rho\right]}\left\{-\left[p_{0}^{2}-\tilde{m}^{2}+\frac{4(\rho-1) t^{2}}{\rho}\right]\left(|p \cdot J|^{2}-p_{0}^{2}\left|J_{\perp}\right|^{2}\right)+p_{0}^{2}\left|J_{z}\right|^{2}\left[p_{0}^{2}-\tilde{m}^{2}+\frac{\left(\rho^{2}-4\right) t^{2}}{\rho}\right]\right\} .
$$


The residue in the pole $p_{0}^{2}=\tilde{m}^{2}$ gives

$$
\begin{gathered}
\operatorname{Im}\left[\mathscr{R}_{p_{0}^{2}=\tilde{m}^{2}}(\mathcal{S P})\right]=\frac{(\rho+2)}{4}\left|J_{z}\right|^{2} \\
+\frac{(1-\rho)}{(2-\rho)} \frac{1}{\tilde{m}^{2}}\left[|p \cdot J|^{2}-\tilde{m}^{2}\left|J_{\perp}\right|^{2}\right] .
\end{gathered}
$$

It is clear that a nonnegative imaginary part of the residue in the pole $p_{0}^{2}=\tilde{m}^{2}$ depends on the value of $\rho$. In the case of $\rho=1$, we are back to the case $p_{z}=-t$. The possible triple pole for $\rho=2$ and $p_{z}=0$ is not consistent, since the residue diverges. This physical inconsistency of such a case is also related to the stability of the model and will be made clear in the next section.

\section{The Residual Gauge Invariance}

All the above results present us clues of some special feature in the model when $\rho=1$. First, let us rewrite the dispersion relations for the model in a more general way. From the $D$ factor in the denominator, taking $\alpha=1$ and $\mu=1$, we have

$$
\left(p^{2}-\kappa^{2}\right)^{2}+4\left(\kappa^{2} p^{2}-\lambda^{2}\right)=0
$$

which can be factorized as

$$
\left(p^{2}+\kappa^{2}+2 \lambda\right)\left(p^{2}+\kappa^{2}-2 \lambda\right)=0
$$

so that

$$
\omega^{2} \equiv p_{0}^{2}=|p|^{2}-\kappa^{2} \pm 2 \lambda
$$

For the purely spacelike background vector, $\kappa^{\mu}=(0, \vec{\kappa})$, we stay with

$$
\omega^{2}=|p \pm \vec{\kappa}|^{2}
$$

Since the model is massive, we can go to the particle rest frame and get, for these two dispersion relations,

$$
\omega^{2}=|\vec{\kappa}|^{2}
$$

which represents a mode with a positive definite mass which propagates with two degrees of freedom. It is important to emphasize that these results do not depend on the value of the $\rho$ parameter. On the other hand, the $\tilde{D}$ factor is written as

$$
\tilde{D}=\rho \lambda^{2}-\kappa^{2} p^{2}+(1-\rho) \kappa^{4},
$$

which furnishes us the dispersion relation

$$
\omega^{2}=|p|^{2}+\frac{1}{\kappa^{2}}\left[\rho \lambda^{2}+(1-\rho) \kappa^{4}\right]
$$

For the purely spacelike Lorentz-breaking vector, the result is written as

$$
\begin{aligned}
\omega^{2} & =|p|^{2}-\frac{1}{|\vec{\kappa}|^{2}}\left[\rho(p \cdot \vec{\kappa})^{2}+(1-\rho)|\vec{\kappa}|^{4}\right] \\
& =|p|^{2}\left(1-\rho \cos ^{2} \theta\right)-(1-\rho)|\vec{\kappa}|^{2}
\end{aligned}
$$

where $\theta$ is the angle between the vectors $p$ and $\vec{\kappa}$. Let us consider the field propagates in the direction of the Lorentzbreaking vector, such that $\theta=0$. In this situation, we stay with

$$
\omega^{2}=(1-\rho)\left[|p|^{2}-|\vec{\kappa}|^{2}\right],
$$

which imposes some restrictions. First, if $\rho=1$, we have a massless mode. For $\rho \neq 1$, the dispersion relation predicts a massive mode, but with the restriction $\rho>1$, otherwise we have an unstable model, since in the particle rest frame we have $\omega^{2}=(\rho-1)|\vec{\kappa}|^{2}$. However, even with $\rho>1$, the stability of the model imposes severe restrictions, as the magnitude of the field momentum would be limited by that of the background vector, which is very small. The propagation of the field would be practically restricted to the plane orthogonal to $\vec{\kappa}$, although the component of the momentum in the direction of $\vec{\kappa}$ cannot be null (we must have $\rho \neq 2$ ). Thus, in the special case in which $\rho=1$, the model presents a massive mode, $\omega^{2}=|\vec{\kappa}|^{2}$ (coming from the factor $D$ in the denominator), with two degrees of freedom, and a massless mode, which corresponds to a propagation in the direction of the background vector (from $\tilde{D}$ ).

In massive models with violation of gauge symmetry, it is usual to look for hidden symmetries through dualization processes, which could provide an equivalent gaugeinvariant model. The gauge embedding method [72, 73] proved to be effective in this type of procedure in a wide range of models. The approach is based on the transformation of the model in a gauge theory by adding on massshell vanishing terms. This iterative embedding of Noether counterterms is based on the idea of local lifting a global symmetry and is reminiscent to procedures which were important in the construction of component-field supergravity actions [74-76]. It would be interesting to carry out the dualization of the present theory. However, the procedure needs the use of the inverse operator of the mass tensor, $M_{\mu \nu}$ (defined in equation (2)), which is given by

$$
L_{\mu \nu}=\frac{1}{\kappa^{2}}\left\{\eta_{\mu \nu}+\rho \frac{\kappa_{\mu} \kappa_{v}}{(1-\rho) \kappa^{2}}\right\} .
$$

Interestingly, there is no inverse for $M_{\mu \nu}$ when $\rho=1$. This is another compelling clue for the investigation of this particular case.

Last but not least, calculating the one-loop vacuum polarization tensor for the model up to second-order in $\kappa^{\mu}$ 
gives us a transverse result [61], with

$$
\Pi_{\mu \nu}^{(2)}=-\frac{i e^{2}}{6 \pi^{2} m^{2}}\left[1+\frac{2}{5} \frac{p^{2}}{m^{2}}+O\left(\frac{p^{4}}{m^{4}}\right)\right] T_{\mu \nu}
$$

in which $m$ is the fermion mass and

$$
\begin{aligned}
T_{\mu \nu}= & \kappa^{2}\left(p_{\mu} p_{\nu}-p^{2} \eta_{\mu \nu}\right)+p^{2} \kappa_{\mu} \kappa_{v}+(p \cdot \kappa)^{2} \eta_{\mu \nu} \\
& -(p \cdot \kappa)\left(p_{\mu} \kappa_{v}+p_{\nu} \kappa_{\mu}\right) .
\end{aligned}
$$

All these results bring us the possibility of a residual gauge invariance. The gauge-violating term is given by

$$
\mathscr{L}_{M}=\frac{1}{2} A^{\mu} M_{\mu \nu} A^{v}
$$

By considering a gauge transformation on the vectorial field, $A^{\mu}$,

$$
A^{\mu} \longrightarrow A^{\mu}+\partial^{\mu} \chi
$$

we have

$$
\delta \mathscr{L}=A^{\mu} M_{\mu \nu}\left(\partial^{\nu} \chi\right)+\frac{1}{2}\left(\partial^{\mu} \chi\right) M_{\mu \nu}\left(\partial^{\nu} \chi\right)
$$

It is easy to see that if $\partial^{\mu} \chi$ is proportional to $\kappa^{\mu}$, such that $\partial^{\mu} \chi=\beta(z) \kappa^{\mu}$, with $\beta(z)$ a dimensionless function of $z$ (assuming the $z$-axis is parallel to the background vector), we get, explicitly using (2),

$$
\left(\partial^{\mu} \chi\right) M_{\mu \nu}=\beta \kappa^{2} \kappa_{v}(1-\rho)
$$

which is null for $\rho=1$. In this situation, the variation of the Lagrangian is null. Just in order to illustrate, for our purely spacelike vector $\kappa^{\mu}=(0, \vec{\kappa})$, this is accomplished if the gradient of $\chi$ is parallel to $\vec{\kappa}$ in the case $\rho=1$. Thus, there is a class of gauge transformations which leaves the Lagrangian density invariant and, in this sense, we can affirm that the model accommodates a residual gauge invariance when $\rho=1$.

Although we use a specific model in the discussion of the residual gauge invariance, it is easy to see that this is a general result for all the models whose only source of gauge symmetry violation is the above cited $\mathscr{L}_{M}$.

\section{Conclusion}

We studied an alternative CPT-odd electrodynamics which incorporates a general Lorentz-breaking mass term. Actually, it is an extended version of the one obtained from a Palatini-like procedure in [61]. We have showed that the Lorentz-breaking part of the mass tensor affects only one of the three modes of propagation of the gauge field. The $\rho$ parameter that controls this part of the mass tensor plays a fundamental role in preserving the essential physical properties of the model. It was shown, for a spacelike Lorentz- breaking vector $\kappa^{\mu}$, that if $\rho<1$, the model presents serious problems with stability. Moreover, if this parameter is greater than one, a positive definite energy is required, and the field is restricted to propagate in a direction external to a cone defined by the rotation, around the $z$-axis (defined as the direction of the background vector), of the lines $z=$ $\pm\left(t / p_{\perp}\right) u$, being $u$ the axis parallel to the component $\vec{p}_{\perp}$ of the field momentum and $t$ the magnitude of the Lorentzbreaking vector $\vec{\kappa}$. Moreover, the particular case in which $\rho=2$ accommodates a nonphysical triple pole when the component of the field momentum along the $z$-axis is null.

The most interesting case, however, is the one with $\rho=1$, which presents a massless mode of propagation along the $z$ -axis. This fact, together with the lack of an inverse for the mass tensor, led us to investigate the presence of a residual gauge invariance in the model. We then showed that a gauge transformation, $A^{\mu} \longrightarrow A^{\mu}+\partial^{\mu} \chi$, such that $\chi=\chi(z)$, leaves the action unchanged. For a purely spacelike background vector, this corresponds to have the gradiant of $\chi$ parallel to $\vec{\kappa}$. Moreover, this is a general result for models whose only source of gauge symmetry violation is the mass term studied in the present paper.

For future work, it would be interesting to investigate how a dualization process would work in the limit $\rho \longrightarrow 1$. In other words, it would be worth to understand what kind of model comes up in this limit in a more general gaugeinvariant model with $\rho$ undetermined, obtained from a gauge embedding procedure.

\section{Data Availability}

No data were used to support this study.

\section{Conflicts of Interest}

The authors declare that they have no conflicts of interest.

\section{Acknowledgments}

This work was partially supported by Conselho Nacional de Desenvolvimento Cientfico e Tecnológico (CNPq). The authors acknowledge Prof. J. A. Helayël-Neto for elucidating discussions.

\section{References}

[1] V. A. Kostelecký and S. Samuel, "Spontaneous breaking of Lorentz symmetry in string theory," Physical Review D, vol. 39, no. 2, pp. 683-685, 1989.

[2] V. A. Kostelecký and S. Samuel, "Gravitational phenomenology in higher-dimensional theories and strings," Physical Review D, vol. 40, no. 6, pp. 1886-1903, 1989.

[3] S. Doplicher, K. Fredenhagen, and J. E. Roberts, "The quantum structure of spacetime at the Planck scale and quantum fields," Communications in Mathematical Physics, vol. 172, no. 1, pp. 187-220, 1995.

[4] J. Collins, A. Perez, D. Sudarsky, L. Urrutia, and H. Vucetich, "Lorentz invariance and quantum gravity: an additional fine- 
tuning problem?," Physical Review Letters, vol. 93, no. 19, article 191301, 2004.

[5] P. Horava, "Quantum gravity at a Lifshitz point," Physical Review D, vol. 79, no. 8, article 084008, 2009.

[6] S. M. Carroll, G. B. Field, and R. Jackiw, "Limits on a Lorentzand parity-violating modification of electrodynamics," Physical Review D, vol. 41, p. 1231, 1990.

[7] A. P. Baêta Scarpelli, H. Belich, J. L. Boldo, and J. A. HelayëlNeto, "Aspects of causality and unitarity and comments on vortexlike configurations in an Abelian model with a Lorentz-breaking term," Physical Review D, vol. 67, no. 8, article 085021, 2003.

[8] A. P. Baêta Scarpelli and J. A. Helayël-Neto, "Lorentz-violating SO(3) model: discussing unitarity, causality, and 't HooftPolyakov monopoles," Physical Review D, vol. 73, no. 10, article 105020, 2006.

[9] R. Jackiw and V. A. Kosteleckỳ, "Radiatively induced Lorentz and CPT violation in electrodynamics," Physical Review Letters, vol. 82, no. 18, pp. 3572-3575, 1999.

[10] J.-M. Chung and B. K. Chung, "Induced Lorentz- and CPTviolating Chern-Simons term in QED: Fock-Schwinger proper time method," Physical Review D, vol. 63, no. 10, article 105015, 2001.

[11] J.-M. Chung, "Lorentz- and CPT-violating Chern-Simons term in the functional integral formalism," Physical Review $D$, vol. 60, no. 12, article 127901, 1999.

[12] G. Bonneau, "Regularisation: many recipes, but a unique principle: ward identities and normalisation conditions. The case of CPT violation in QED," Nuclear Physics B, vol. 593, no. 12, pp. 398-412, 2001.

[13] M. Perez-Victoria, "Exact calculation of the radiatively induced Lorentz and CPT Violation in QED," Physical Review Letters, vol. 83, no. 13, pp. 2518-2521, 1999.

[14] J. High, "Motion on moduli spaces with potentials," Journal of High Energy Physics, vol. 2001, no. 12, p. 032, 2001.

[15] O. A. Battistel and G. Dallabona, "Role of ambiguities and gauge invariance in the calculation of the radiatively induced Chern-Simons shift in extended QED," Nuclear Physics B, vol. 610, no. 1-2, pp. 316-330, 2001.

[16] O. A. Battistel and G. Dallabona, "Ambiguities versus gauge invariance in the calculation of the radiatively induced Chern-Simons shift in extended QED," Journal of Physics G, vol. 27, no. 7, pp. L53-L59, 2001.

[17] O. A. Battistel and G. Dallabona, "Consistency in perturbative calculations and radiatively induced Lorentz and CPT violations," Journal of Physics G, vol. 28, no. 8, pp. L23-L32, 2002.

[18] A. P. Baêta Scarpelli, M. Sampaio, M. C. Nemes, and B. Hiller, "Chiral anomaly andCPTinvariance in an implicit momentum space regularization framework," Physical Review D, vol. 64, no. 4, article 046013, 2001.

[19] A. P. Baêta Scarpelli, M. Sampaio, M. C. Nemes, and B. Hiller, "Gauge invariance and the CPT and Lorentz violating induced Chern-Simons-like term in extended QED," The European Physical Journal C, vol. 56, no. 4, pp. 571-578, 2008.

[20] C. Adam and F. R. Klinkhamer, "Causality and CPT violation from an Abelian Chern-Simons-like term," Nuclear Physics B, vol. 607, no. 1-2, pp. 247-267, 2001.

[21] C. Adam and F. R. Klinkhamer, "Photon decay in a CPTviolating extension of quantum electrodynamics," Nuclear Physics B, vol. 657, pp. 214-228, 2003.
[22] A. A. Andrianov and R. Soldati, "Lorentz symmetry breaking in Abelian vector-field models with Wess-Zumino interaction," Physical Review D, vol. 51, no. 10, pp. 5961-5964, 1995.

[23] A. A. Andrianov and R. Soldati, "Patterns of Lorentz symmetry breaking in QED by CPT-odd interaction," Physics Letters B, vol. 435, no. 3-4, pp. 449-452, 1998.

[24] A. A. Andrianov, R. Soldati, and L. Sorbo, "Dynamical Lorentz symmetry breaking from a(3+1)-dimensional axion-WessZumino model," Physical Review D, vol. 59, no. 2, article 025002, 1998.

[25] A. A. Andrianov, D. Espriu, P. Giacconi, and R. Soldati, "Anomalous positron excess from Lorentz-violating QED," Journal of High Energy Physics, vol. 909, p. 057, 2009.

[26] J. Alfaro, A. A. Andrianov, M. Cambiaso, P. Giacconi, and R. Soldati, "Bare and induced lorentz and cpt invariance violations in qed," International Journal of Modern Physics A, vol. 25, no. 16, pp. 3271-3306, 2010.

[27] V. C. Zhukovsky, A. E. Lobanov, and E. M. Murchikova, "Radiative effects in the standard model extension," Physical Review D, vol. 73, no. 6, article 065016, 2006.

[28] D. Colladay and V. A. Kostelecky, "CPT violation and the standard model," Physical Review D, vol. 55, p. 6760, 1997.

[29] D. Colladay and V. A. Kostelecky, "Lorentz-violating extension of the standard model," Physical Review D, vol. 58, article $116002,1998$.

[30] S. Coleman and S. L. Glashow, "Cosmic ray and neutrino tests of special relativity," Physical Review D, vol. 405, no. 3-4, pp. 249-252, 1997.

[31] S. Coleman and S. L. Glashow, "High-energy tests of Lorentz invariance," Physical Review D, vol. 59, no. 11, article 116008, 1999.

[32] V. A. Kosteleckỳ and N. Russell, "Data tables for Lorentz and CPT violation," Reviews of Modern Physics, vol. 83, no. 1, pp. 11-31, 2011.

[33] P. Robles and F. Claro, "Can there be massive photons? A pedagogical glance at the origin of mass," European Journal of Physics, vol. 33, p. 1217, 2012.

[34] L. C. Tu, J. Luo, and G. T. Gillies, "The mass of the photon," Reports on Progress in Physics, vol. 68, no. 1, pp. 77-130, 2005.

[35] A. S. Goldhaber and M. M. Nieto, "Photon and graviton mass limits," Reviews of Modern Physics, vol. 82, p. 939, 2010.

[36] G. Spavieri, J. Quintero, G. T. Gillies, and M. Rodriguez, "Atomic, molecular, optical and plasma physics," European Physical Journal D, vol. 61, p. 531, 2011.

[37] M. V. S. Fonseca and A. V. Paredes, "Is it possible to accommodate massive photons in the framework of a gaugeinvariant electrodynamics?," Brazilian Journal of Physics, vol. 40, p. 319, 2010.

[38] J.-J. Wei and X.-F. Wu, "Robust limits on photon mass from statistical samples of extragalactic radio pulsars," Journal of Cosmology and Astroparticle Physics, vol. 2018, no. 7, p. 045, 2018.

[39] L. Shao and B. Zhang, "Bayesian framework to constrain the photon mass with a catalog of fast radio bursts," Physical Review D, vol. 95, no. 12, article 123010, 2017.

[40] L. Bonetti, J. Ellis, N. E. Mavromatos, A. S. Sakharov, E. K. Sarkisyan-Grinbaum, and A. D. A. M. Spallicci, "FRB 121102 casts new light on the photon mass," Physics Letters B, vol. 768, pp. 326-329, 2017.

[41] Y.-P. Yang and B. Zhang, "Tight constraint on photon mass from pulsar spindown," The Astrophysical Journal, vol. 842, no. 1, p. 23, 2017. 
[42] J.-J. Wei, E.-K. Zhang, S.-B. Zhang, and X.-F. Wu, "New limits on the photon mass with radio pulsars in the Magellanic Clouds," Research in Astronomy and Astrophysics, vol. 17, no. 2, p. 13, 2017.

[43] B. Zhang, Y.-T. Chai, Y.-C. Zou, and X.-F. Wu, "Constraining the mass of the photon with gamma-ray bursts," Journal of High Energy Astrophysics, vol. 11-12, pp. 20-28, 2016.

[44] L. Bonetti, J. Ellis, N. E. Mavromatos, A. S. Sakharov, E. K. G. Sarkisyan-Grinbaum, and A. D. A. M. Spallicci, "Photon mass limits from fast radio bursts," Physics Letters B, vol. 757, pp. 548-552, 2016.

[45] X. F. Wu, S. B. Zhang, H. Gao et al., "Constraints on the photon mass with fast radio bursts," The Astrophysical Journal Letters, vol. 822, p. L15, 2016.

[46] A. Retinò, A. D. A. M. Spallicci, and A. Vaivads, "Solar wind test of the de Broglie-Proca massive photon with Cluster multi-spacecraft data," Astroparticle Physics, vol. 82, p. 49, 2016.

[47] Particle Data Group, "Review of particle physics," Physical Review D, vol. 98, no. 3, article 030001, 2018.

[48] D. D. Ryutov, "The role of finite photon mass in magnetohydrodynamics of space plasmas," Plasma Physics and Controlled Fusion, vol. 39, no. 5A, pp. A73-A82, 1997.

[49] D. D. Ryutov, "Using plasma physics to weigh the photon," Plasma Physics and Controlled Fusion, vol. 49, no. 12B, pp. B429-B438, 2007.

[50] L. Davis, A. S. Goldhaber, and M. M. Nieto, "Limit on the photon mass deduced from Pioneer-10 observations of Jupiter's magnetic field," Physical Review Letters, vol. 35, p. 1402, 1975.

[51] G. Chibisov, "Astrophysical upper limits on the photon rest mass," Uspekhi Fizicheskikh Nauk, vol. 119, no. 7, p. 551, 1976.

[52] L. Bonetti, L. R. dos Santos Filho, J. A. Helayël-Neto, and A. D. A. M. Spallicci, "Effective photon mass by Super and Lorentz symmetry breaking," Physics Letters B, vol. 764, pp. 203-206, 2017.

[53] L. Bonetti, L. R. dos Santos, J. A. Helayël-Neto, and A. D. A. M. Spallicci, "Photon sector analysis of Super and Lorentz symmetry breaking: effective photon mass, bi-refringence and dissipation," European Physical Journal C: Particles and Fields, vol. 78, no. 10, p. 811, 2018.

[54] M. M. Ferreira, J. A. Helayël-Neto, C. M. Reyes, M. Schreck, and P. D. S. Silva, "Unitarity in Stückelberg electrodynamics modified by a Carroll-Field-Jackiw term," Physics Letters B, vol. 804, article 135379, 2020.

[55] G. Gabadadze and L. Grisa, "Lorentz-violating massive gauge and gravitational fields," Physics Letters B, vol. 617, p. 124, 2005.

[56] G. Dvali, M. Papucci, and M. D. Schwartz, "Infrared Lorentz violation and slowly instantaneous electricity," Physical Review Letters, vol. 94, article 191602, 2005.

[57] B. Altschul, "Radiatively induced Lorentz-violating photon masses," Physical Review D, vol. 73, article 036005, 2006.

[58] M. Cambiaso, R. Lehnert, and R. Potting, "Massive photons and Lorentz violation," Physical Review D, vol. 85, article 085023, 2012.

[59] B. Altschul, "Lorentz violation and the Higgs mechanism," Physical Review D, vol. 86, article 045008, 2012.

[60] H. G. Fargnoli, L. C. T. Brito, A. P. Baêta Scarpelli, and M. Sampaio, "Dual embedding of extended models with a Lorentz-breaking mass term," Physical Review D, vol. 90, no. 8, article 085016, 2014.
[61] J. C. C. Felipe, H. G. Fargnoli, A. P. Baêta Scarpelli, and L. C. T. Brito, "Consistency of an alternative CPT-odd and Lorentzviolating extension of QED," International Journal of Modern Physics A, vol. 34, no. 25, article 1950139, 2019.

[62] D. J. Toms"Quantization of the minimal and non-minimal vector field in curved space,” http://arxiv.org/abs/:1509.05989.

[63] I. L. Buchbinder, T. P. Netto, and I. L. Shapiro, "Massive vector field on curved background: nonminimal coupling, quantization, and divergences," Physical Review D, vol. 95, no. 8, article 085009, 2017.

[64] M. S. Ruf and C. F. Steinwachs, "Renormalization of generalized vector field models in curved spacetime," Physical Review D, vol. 98, article 025009, 2018.

[65] C. Garcia-Recio and L. L. Salcedo, "Renormalization of vector fields with mass-like coupling in curved spacetime," European Physical Journal C: Particles and Fields, vol. 79, no. 5, p. 438, 2019.

[66] E. C. G. Stueckelberg, "Interaction energy in electrodynamics and in the field theory of nuclear forces," Helvetica Physica Acta, vol. 11, pp. 225-244, 1938.

[67] E. C. G. Stueckelberg, "Forces of interaction in electrodynamics and in the field theory of nuclear forces.(parts ii and iii)," Helvetica Physica Acta, vol. 11, p. 299, 1938.

[68] B. Körs and P. Nath, "A Stueckelberg extension of the standard model," Physics Letters B, vol. 586, p. 366, 2004.

[69] H. Ruegg and M. Ruiz-Altaba, "The stueckelberg field," International Journal of Modern Physics A, vol. 19, pp. 3265-3347, 2004.

[70] R. Casana, M. M. Ferreira Jr., and R. P. M. Moreira, “Consistency analysis of a nonbirefringent Lorentz-violating planar model," The European Physical Journal C, vol. 72, article 2070, 2012.

[71] M. Veltman, "Quantum theory of gravitation," in Methods in Field Theory, R. Bailian and J. Zinn-Justin, Eds., North-Holland Publising Company and World Scientific Publising Co Ltd, Singapore, 1981.

[72] A. Ilha and C. Wotzasek, "Duality equivalence between selfdual and topologically massive non-abelian models," Nuclear Physics B, vol. 604, p. 426, 2001.

[73] M. A. Anacleto, A. Ilha, J. R. S. Nascimento, R. F. Ribeiro, and C. Wotzasek, "Dual equivalence between self-dual and Maxwell-Chern-Simons models coupled to dynamical U(1) charged matter," Physics Letters B, vol. 504, p. 268, 2001.

[74] D. Z. Freedman, P. van Nieuwenhuizen, and S. Ferrara, "Progress toward a theory of supergravity," Physical Review D, vol. 13, no. 12, pp. 3214-3218, 1976.

[75] S. Ferrara, D. Z. Freedman, P. van Nieuwenhuizen, P. Breitenlohner, F. Gliozzi, and J. Scherk, "Scalar multiplet coupled to supergravity," Physical Review D, vol. 15, no. 4, pp. 1013-1018, 1977.

[76] S. Ferrara, J. Scherk, and P. van Nieuwenhuizen, "Locally Supersymmetric Maxwell-Einstein Theory," Physical Review Letters, vol. 37, no. 16, pp. 1035-1037, 1976. 